\title{
STRUCTURAL AND SOCIAL ENRICHMENT: EFFECTS ON THE MORPHOLOGY OF A TROPICAL HATCHERY FISH
}

\author{
SARAIVA, S. O. ${ }^{{ }^{*}}-$ POMPEU, P. S. ${ }^{1}$ \\ ${ }^{1}$ Universidade Federal de Lavras \\ Setor de Ecologia, Departamento de Biologia, P. O. Box 3037, CEP 37200- 000, Lavras MG, \\ Brazil \\ (phone: +55-35-3829-1914; fax: +55-35-3829-1341) \\ *Corresponding author \\ e-mail: sarahsaraiva@hotmail.com; phone: +55-37-99999-9459
}

(Received $7^{\text {th }}$ Jan 2016; accepted $23^{\text {rd }}$ Apr 2016)

\begin{abstract}
Hatchery tanks offer conditions completely different from the natural environment, with a high degree of environmental homogeneity. As a result, for some species, farmed fish can be morphologically distinct from their wild counterparts and the populations can be morphologically less diverse. To minimize the effects of the homogeneity, the environmental enrichment of tanks can be proposed as a way of resembling natural conditions. In this study we evaluated two types of enrichment: structural, which was performed by adding submerged logs (tree trunks) and artificial aquatic plants to increase environmental complexity; and social, which was accomplished by the simultaneous farming of two species in one pond to increase the degree of ecological interactions. The aim was to test whether social and structural enrichment of hatchery tanks modifies the morphology and anatomical variability of curimba Prochilodus lineatus. The results showed that both types of enrichment led to morphological divergence, however social enrichment had a bigger effect, increasing morphological variability and diluting the effect of structural enrichment. Therefore, the simultaneous farming of two or more species can constitute a simple tool for hatchery fish management, to increase population morphological variability and potentially increase the chances for survival after release into the natural environment.
\end{abstract}

Keywords: environmental homogeneity; fish stocking; morphological variability; social interactions; structural complexity

\section{Introduction}

Fish stocking consists of breeding fish in captivity (in hatcheries) for subsequent release into aquatic environments to enhance native populations. This management method has been long used worldwide to mitigate the negative effects from habitat loss and environmental disturbances, such as the construction of hydroelectric dams. However, the low fishery yield and the precarious conservation status of native migratory populations in southern and southeastern Brazil indicate that this practice has not been satisfactory (Agostinho et al., 2007). The poor stocking efficiency can be largely explained by low survival rates after release (Maynard et al., 1994), as the released fish are more adapted to captive conditions and consequently ill-suited to live in the wild (Olla et al., 1998; Araki et al., 2008; Belk et al., 2008; Johnsson et al., 2014). Hatchery tanks are quite homogenous and presents high density and less variable population, lacking of predators and prey, but constant food availability by pellets, while in the wild variables are spatially and temporally unpredictable, including turbidity level, water flow and structural complexity provided by gravel, rocks, plants and trees (Johnsson et al., 2014). As a result, farmed fish differ from wild fish in several aspects, like morphology, exhibiting distinct colorations (Maynard et al., 1994), different body, head and fin proportions (Swain et al., 1991; Flemming et al., 1994; 
Hard et al., 2000; Solem et al., 2006; Belk et al., 2008) and less morphological variability (Taylor, 1986; Saraiva and Pompeu, 2016).

Thus, the environmental enrichment of hatchery tanks has been proposed as a means of minimizing the effects of captivity on the morphology of farmed fish (Näslund and Johnsson, 2014). The aim of enrichment is to modify captive conditions to improve biological functioning (Newberry, 1995). These modifications can be of various types, including the addition of sensory stimuli, greater complexity and increased ecological interaction (Batzina and Karakatsouli, 2012). Generally, all types of enrichment seek to make the conditions of captivity more similar to those of the natural environment, increasing animal wellbeing and, in the case of fish stocking, increasing the chances of survival after release into the natural environment.

The addition of shelter and substrates to the captive environment is called structural enrichment. Some studies have evaluated the effects of this kind of enrichment on farmed fish behavior (Berejikian et al., 2001, 2000; Brown et al., 2003), growth (Batzina and Karakatsouli, 2012), predation risk (Roberts et al., 2011) and morphology (Garduño-Paz et al., 2010; Saraiva and Pompeu, 2014). The increase of ecological interactions is called social enrichment, which involves the opportunity for hatchery individuals to live together with other conspecifics or individuals of a different species. The coexistence of two or more species can generate interspecific competition, which greatly affects individuals and can reduce their fertility, survival and growth (Begon et al., 2007). Social enrichment has been little studied in fish, but has been evaluated for other animal groups such as mammals (Schapiro et al., 1996; Elliott and Grunberg, 2005; Leonardi et al., 2010).

Fish hatchery tanks are most commonly simple environments lacking shelter, substrates or obstacles, and with a single species. By contrast, natural environments are structurally complex and contain many species sharing the same habitat and competing for food and shelter. In the present study we farmed two tropical characiform fishes, curimba Prochilodus lineatus (Valenciennes, 1837) and piapara Leporinus elongatus Valenciennes, 1837, simultaneously in structured tanks containing logs and artificial aquatic plants to assess whether social and structural enrichment affect fish morphology.

Curimba and piapara are two migratory fish species native from the Paraná river basin that are commonly used in fish stocking. In the wild, they generally share the same oxbow lake environment during their early development (Agostinho and JúlioJunior, 1999). In fish hatcheries, these two species, like any others that share a tank, can be considered competitive as they vie for space and food. In structured tanks, they may also compete for the substrate and shelter provided by the structures. Curimba was chosen as the target species of the present study as it is the main species used for stocking enhancement on Fish Station of Volta Grande, on Parana river, due to its easy management in hatcheries.

We assumed that piapara would compete with curimba thereby increasing social complexity, and that the presence of logs and artificial macrophytes in the tanks would increase structural complexity. These two forms of enrichment together would make the hatchery environment more similar to a natural one. Thus, our hypothesis was that curimba farmed in the enriched tanks would exhibit different and more varied morphology than those farmed in conventional tanks. 


\section{Material and Methods}

\section{Breeding}

The experiment was conducted at the fish hatchery station of the Volta Grande hydroelectric dam in Conceição das Alagoas, Minas Gerais State, Brazil. In December 2011, three female and six male curimba and two female and three male piapara, already predisposed for breeding, were selected from the available breeding stocks. Breeding for both species was conducted similarly and simultaneously, although in separate aquariums.

Spawning was induced by the application of three doses of crude catfish pituitary extract (Ictalurus punctatus from North America), calculated according to the weight of each individual. The newly fertilized eggs from all the progenitors of each species were pooled, homogenized, and transferred to incubators at equal densities. Three days after hatching, the contents of each incubator were transferred to a tank. The density of the postlarvae that were introduced to each tank was controlled during the egg stage based on the calculated fertilization rate (eggs hatched per milliliter). Using this rate, it was possible to determine the number of viable eggs per milliliter and, therefore, the volume of eggs required to generate the density of postlarvae specified for each tank. Each tank received a density of 150 larvae per square meter. This density is 50\% less than the density normally used at Volta Grande Station and was chosen to favor the growth and survival of fish and also to provide at least 50 curimba in each tank at the end of the experiment.

\section{Treatments}

Eight fish hatchery tanks ( $3 \mathrm{~m} \times 2.83 \mathrm{~m} \times 0.75 \mathrm{~m})$ with concrete walls and earthen floors were used. To test social enrichment only curimba were farmed in four tanks, while curimba and piapara were farmed together in the remaining tanks. To test structural enrichment we used two tanks without structures and the other six were structured using three different designs (Fig. 1). Each tank represented one of the following treatments:

1. C: control tank with curimba and without structures;

2. L: tank with curimba and logs (tree trunks);

3. M: tank with curimba and artificial macrophytes;

4. B: tank with curimba and both types of structures, logs and macrophytes;

5. P: tank with curimba and piapara, but without structures;

6. PL: tank with curimba, piapara and logs;

7. PM: tank with curimba, piapara and artificial macrophytes;

8. PB: tank with curimba, piapara and both logs and macrophytes.

Logistical problems prevented us to replicate the treatments. However, previous study at the same hatchery and using the same tanks and the same types of structural enrichment have indicated that there was no significant effect of the tank on the morphology of fish (Saraiva and Pompeu, 2014)

In tanks with piapara, to maintain the fish density of $150 / \mathrm{m}^{2}$, postlarvae were added at a ratio of 100 curimba to 50 piapara per square meter. All the postlarvae were added to the tanks on a single day (in December 2011), three days after hatching. The tanks were structured one month after adding the fish (in January 2012), when the fish were one month old. During the experiment fish were fed once a day with commercial pellets for tropical fish (52\% crude protein) and all tanks received the same amount. 

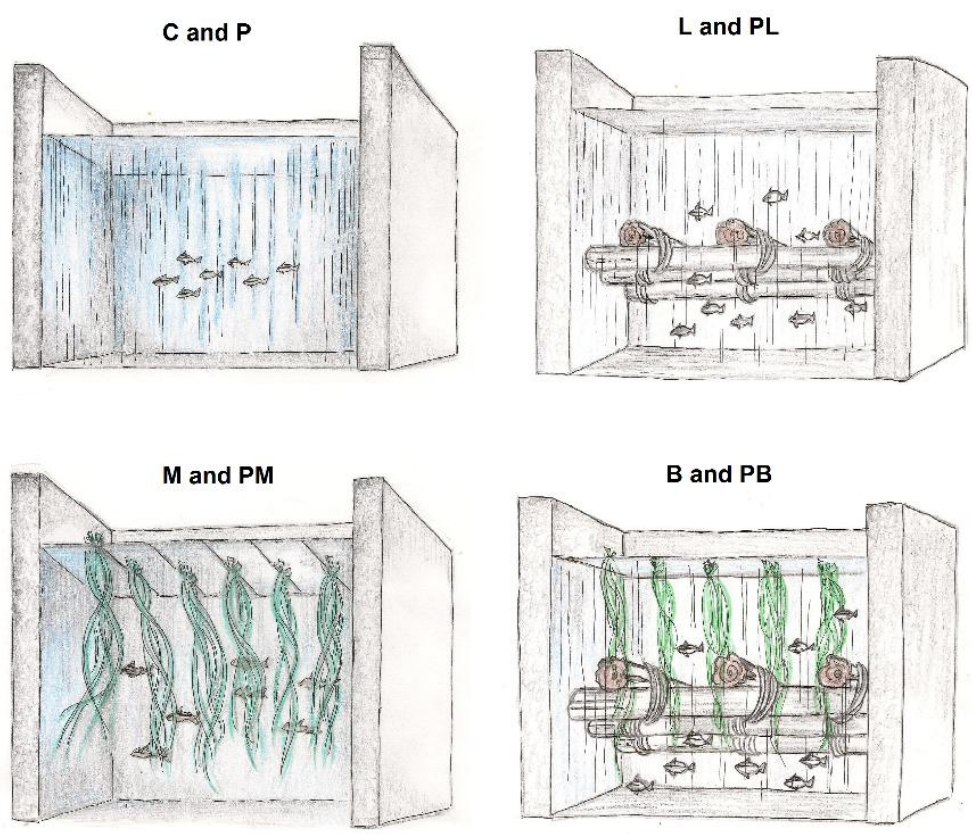

Figure 1. Schematic drawing of the four types of tanks used to evaluate the structural enrichment: $(C$ and $P)$ control, the tank did not receive any type of structuring; ( $L$ and $P L)$ logs, the tank was structured with six eucalyptus tree logs, set in the middle of the tank so that they were submersed after the tank was filled. Three of the logs were arranged along the tank and the other three were arranged perpendicular; ( $M$ and $P M)$ artificial macrophytes, the tank received plastic macrophytes hung on a kind of clothesline placed above the tank, so that the plastic filaments came in contact with the water. Each tank received twenty five plastic macrophytes, arranged in five parallel rows of five plants each; ( $B$ and $P B)$ logs and artificial macrophytes, the tank received both types of structures, submersed logs and artificial macrophytes.

\section{Collection}

The collections were made in March of 2012, when the fish were three months old and had been exposed to the treatments for two months. To capture fish, the water level in the tanks was lowered and dip nets were used. All curimba in each tank were captured and the piapara were kept alive in tanks for use by the Volta Grande fish hatchery station. After collection, the curimba were anesthetized with clove oil, fixed in $10 \%$ formaldehyde and subsequently preserved in $70 \%$ alcohol. The fish voucher specimens were deposited in the Ichthyological Collection of the Federal University of Lavras (CI-UFLA 786-787).

\section{Morphometry}

All fish collected were measured and weighed in a laboratory. Twenty-seven morphometric measurements were taken from each of the individuals collected (standard length, SL; body height, BH; body width, BW; mean body height, $\mathrm{MH}$; body area, BA; head length, HeL; head height, $\mathrm{HeH}$; eye height, EH; eye area, EA; length of the caudal peduncle, LCdP; height of the caudal peduncle, HCdP; width of the caudal peduncle, WCdP; length of the dorsal fin, LDsF; height of the dorsal fin, HDsF; area of 
the dorsal fin, ADsF; length of the caudal fin, LCdF; height of the caudal fin, $\mathrm{HCdF}$; area of the caudal fin, ACdF; length of the anal fin, LAnF; width of the anal fin, WAnF; area of the anal fin, AAnF; length of the pelvic fin, LPIF; width of the pelvic fin, WPIF; area of the pelvic fin, APIF; length of the pectoral fin, LPtF; width of the pectoral fin, WPtF; area of the pectoral fin, APtF). Linear measurements were obtained directly from the body of the fish, always on the left side and by the same person, using a digital caliper accurate to $0.01 \mathrm{~mm}$. Areas were measured by drawing an outline of the body and fins of each fish on paper. The drawings were then scanned and the areas were calculated using the Image $\mathbf{J}$ software (U. S. National Institutes of Health, Bethesda, Maryland, USA). From the morphometric measurements, twenty-one ecomorphological traits (compression index, $\mathrm{CI}=\mathrm{BH} / \mathrm{BW}$; index of ventral flattening, $\mathrm{IVF}=\mathrm{MH} / \mathrm{BH}$; relative body height, $\mathrm{RBH}=\mathrm{BH} / \mathrm{SL}$; relative eye position, $\mathrm{REP}=\mathrm{EH} / \mathrm{HeH}$; relative eye area, $\mathrm{REA}=\mathrm{EA} /(\mathrm{SL})^{2}$; relative head length, $\mathrm{RHeL}=\mathrm{HeL} / \mathrm{SL}$; relative length of the caudal peduncle, RLCdP $=\mathrm{LCdP} / \mathrm{SL}$; relative height of the caudal peduncle, $\mathrm{RHCdP}=$ $\mathrm{HCdP} / \mathrm{BH}$; relative width of the caudal peduncle, $\mathrm{RWCdP}=\mathrm{WCdP} / \mathrm{BW}$; compression index of the caudal peduncle, $\mathrm{CICdP}=\mathrm{HCdP} / \mathrm{WCdP}$; relative area of the dorsal fin, $\mathrm{RADsF}=\mathrm{ADsF} / \mathrm{BA}$; relative area of the caudal fin, $\mathrm{RACdF}=\mathrm{ACdF} / \mathrm{BA}$; Aspect ratio of the caudal fin, $\mathrm{ARCdF}=(\mathrm{HCdF})^{2} / \mathrm{ACdF}$; relative area of the anal fin, $\mathrm{RAAnF}=$ $\mathrm{AAnF} /(\mathrm{SL})^{2}$; aspect ratio of the anal fin, $\mathrm{ARAnF}=(\mathrm{LAnF})^{2} / \mathrm{AAnF}$; relative length of the pelvic fin, $\mathrm{RLPIF}=\mathrm{LPIF} / \mathrm{SL}$; relative area of the pelvic fin, $\mathrm{RAPIF}=\mathrm{APIF} / \mathrm{BA}$; aspect ratio of the pelvic fin, $\mathrm{ARPIF}=\mathrm{LPIF} / \mathrm{WPIF}$; relative length of the pectoral fin, $\mathrm{RLPtF}=\mathrm{LPtF} / \mathrm{SL}$; relative area of the pectoral fin, $\mathrm{RAPtF}=\mathrm{APtF} / \mathrm{BA}$; aspect ratio of the pectoral fin, $\mathrm{ARPtF}=\mathrm{LPtF} / \mathrm{WPtF}$ ) were calculated according to Hora (1930); Gosline (1971); Gatz (1979); Watson and Balon (1984); Winemiller (1991); Pouilly et al. (2003); Oliveira \& Goulart (2005). Each individual was considered a sampling unit of its related treatment.

\section{Statistical analyses}

To evaluate whether tank enrichment affects the growth and nutritional status of fish, we compared the standard lengths and the condition factors of the individuals from different treatments. The standard lengths were compared using ANOVA and Tukey's test. The condition factors (CFs) were calculated using the formula:

$$
C F=\frac{W}{(S L)^{b}}
$$

where $\mathrm{W}$ is the weight, SL is the standard length, and $b$ is the slope of the regression line between the weight and the length. This metric is based on the assumption that individuals of a given length with a greater mass are in better condition than those with a lesser mass (Ricker, 1975). Having established the normality of the data, the condition factors were compared between treatments by ANOVA and Tukey's test.

Principal components analysis (PCA) were used to determine the distribution of individuals in the morphological space. Discriminant analysis (DCA) was performed to assess morphological differences and the Squared Mahalanobis Distances were considered to identify the degree of differentiation among treatments due to social and structural enrichments. 
Changes in morphological variability were sought by the distance of each individual from the centroid of its population (CD), which was calculated from distance matrices. The CD metric is one estimate of the relative size of the morphological hypervolume occupied by the population (Winemiller, 1991), which reflects the morphological variability of the population. An ANOVA with Tukey's post hoc test was performed on the calculated $C D$ values to assess possible differences in morphological variability among the populations under each treatment.

\section{Results}

The number of curimba at the end of the experiment differed among tanks $(\mathrm{C}=50$, $\mathrm{L}=51, \mathrm{M}=22, \mathrm{~B}=34, \mathrm{P}=48, \mathrm{PL}=46, \mathrm{PM}=15$ and $\mathrm{PB}=27$ ) and was substantially lower in the tanks enriched with macrophytes (M, B, PM and PB), despite the same post-larvae density introduced at the beginning of the experiment. This indicates a higher mortality rate in tanks with artificial macrophytes, which was even higher than those tanks with piapara. On the other hand, enrichment with logs and/or piapara (i.e. without macrophytes) did not affect curimba survival. The number of piapara left in each tank at the end of the experiment was fairly similar $(\mathrm{P}=19, \mathrm{PL}=20, \mathrm{PM}=22, \mathrm{~PB}=20)$.

Average standard length differed between treatments (ANOVA $-\mathrm{F}(7 ; 284)=2.696 ; \mathrm{p}=$ 0.0102). Fish from all enrichment treatments presented higher size averages than the control (C), however Tukey's test revealed that differences were only significant within tanks PL, PM and PB (Fig. 2a).

The condition factor (CF) also varied depending on the enrichment type (Fig. $2 b$ ). Fish with the best condition were from tanks L and B, indicating that the presence of logs in tanks improved the physiological status of fish. However, this effect only occurred when curimba were farmed alone. When piapara were also present, the CF was lower and did not differ from the control (C).
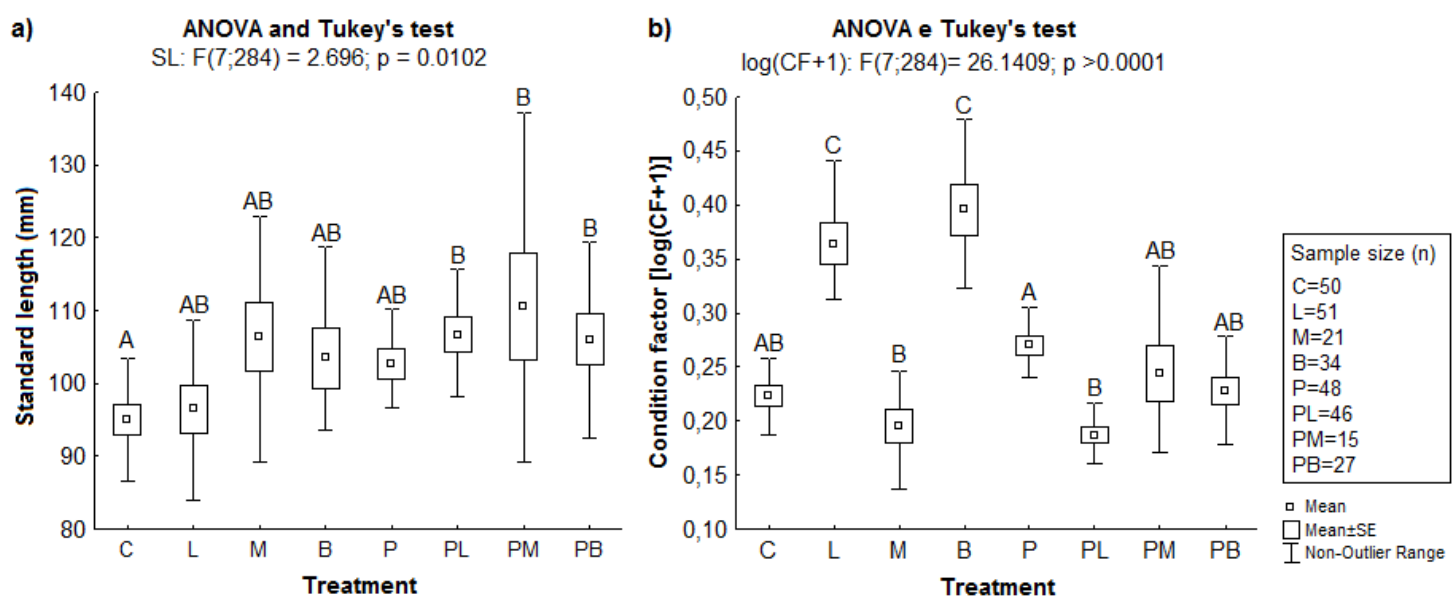

Figure 2. a) Curimba standard length per treatment. After a significant ANOVA $(P<0.05)$, Tukey's test compared standard length averages among treatments. b) Log of the curimba condition factor plus one $[\log (C F+1)]$ for each treatment. After a significant ANOVA $(p<$ $0.05)$, Tukey's test compared the log $(C F+1)$ values averages among the treatments $(C=$ control tank, $L=$ tank with logs, $M=$ tank with artificial macrophytes, $B=$ tank with both logs and artificial macrophytes, $P=$ tank with piapara, $P L=$ tank with piapara and logs, $P M=$ tank with piapara and artificial macrophytes and $P B=$ tank with piapara and both logs and artificial macrophytes). Treatments with the same letter above their ranges did not differ significantly. 
With respect to morphology, the curimba populations of each treatment occupied distinct morphological spaces with little overlap between the tanks with and without piapara, according to PCA (Fig. $3 a$ ). The first two PCA axes accounted for $39.59 \%$ of the variance: the first axis represented $22.76 \%$ and the second axis $16.83 \%$. The contributions of the variables to the PCA axes (Table 1) showed that RADsF, RACdF, RAAnF and RAPIF positively influenced the first axis, whereas CI negatively influenced the second axis. Analyzing PCA separately for the treatments with and without piapara, stays clear that overlap was bigger when piapara was present (Figs. 3b$c)$. In the absence of piapara the first two PCA axes accounted for $41.83 \%$ of the variance: the first axis represented $24.81 \%$ and the second axis $17.02 \%$. The traits that most contributed for the differentiation among treatments in the first axis were CI, REA, RHeL (positively) and RHCdP (negatively) and in the second axis were RAPIF and RAPtF (positively). In the presence of piapara the first two PCA axes accounted for $28.58 \%$ of the variance: the first axis represented $19.90 \%$ and the second axis $8.68 \%$. The traits that most contributed for the differentiation among treatments were $\mathrm{CI}$ and RHeL, both positively and in the first axis.

Table 1. Contributions of ecomorphological traits to the first two axes of the principal component analysis for the treatments without and with pipara, analyzed together and separately. Highlighted in bold are the most important traits for differentiation among treatments in each axis.

\begin{tabular}{l|c|c|c|c|c|c}
\hline \multirow{2}{*}{ Trait } & \multicolumn{2}{|c|}{$\begin{array}{c}\text { With and without } \\
\text { piapara }\end{array}$} & \multicolumn{2}{c|}{ Without piapara } & \multicolumn{2}{c}{ With pipara } \\
\cline { 2 - 7 } & PCA 1 & PCA 2 & PCA 1 & PCA 2 & PCA 1 & PCA 2 \\
\hline CI & $-0,10$ & $\mathbf{- 0 , 8 3}$ & $\mathbf{0 , 8 3}$ & $-0,29$ & $\mathbf{0 , 8 1}$ & $-0,10$ \\
IVF & 0,04 & 0,31 & $-0,45$ & $-0,14$ & 0,05 & 0,01 \\
RBH & $-0,22$ & $-0,41$ & 0,26 & $-0,18$ & 0,52 & $-0,17$ \\
REP & $-0,17$ & $-0,43$ & 0,41 & $-0,11$ & 0,35 & 0,01 \\
REA & $-0,02$ & $-0,34$ & $\mathbf{0 , 8 6}$ & $-0,06$ & 0,11 & $-0,25$ \\
RHeL & 0,08 & $-0,77$ & $\mathbf{0 , 8 7}$ & $-0,03$ & $\mathbf{0 , 8 1}$ & 0,00 \\
RLCdP & 0,38 & 0,31 & $-0,34$ & 0,19 & $-0,16$ & 0,14 \\
RHCdP & 0,15 & 0,68 & $\mathbf{- 0 , 8 1}$ & 0,22 & $-0,70$ & 0,19 \\
RWCdP & $-0,20$ & $-0,10$ & $-0,31$ & $-0,16$ & $-0,09$ & 0,58 \\
CICdP & 0,15 & $-0,30$ & 0,65 & 0,04 & 0,47 & $-0,48$ \\
RADsF & $\mathbf{0 , 8 9}$ & $-0,16$ & 0,37 & 0,47 & 0,48 & 0,47 \\
RACdF & $\mathbf{0 , 8 8}$ & $-0,05$ & 0,13 & 0,61 & $-0,04$ & 0,31 \\
ARCdF & $-0,57$ & 0,15 & $-0,31$ & $-0,32$ & 0,07 & 0,06 \\
RAAnF & $\mathbf{0 , 8 9}$ & $-0,10$ & 0,18 & 0,77 & 0,24 & 0,47 \\
ARAnF & $-0,70$ & $-0,14$ & $-0,04$ & $-0,64$ & 0,10 & $-0,31$ \\
RLPIF & 0,00 & $-0,66$ & 0,62 & 0,30 & 0,70 & 0,18 \\
RAPIF & $\mathbf{0 , 8 6}$ & $-0,03$ & $-0,10$ & $\mathbf{0 , 8 8}$ & 0,33 & $-0,16$ \\
ARPIF & 0,07 & $-0,46$ & 0,62 & 0,05 & 0,46 & 0,24 \\
RLPtF & 0,08 & $-0,52$ & 0,35 & 0,27 & 0,69 & 0,14 \\
RAPtF & 0,38 & 0,10 & $-0,27$ & $\mathbf{0 , 8 1}$ & 0,25 & $-0,22$ \\
ARPtF & 0,61 & $-0,14$ & 0,12 & $-0,05$ & 0,20 & 0,52 \\
\hline
\end{tabular}



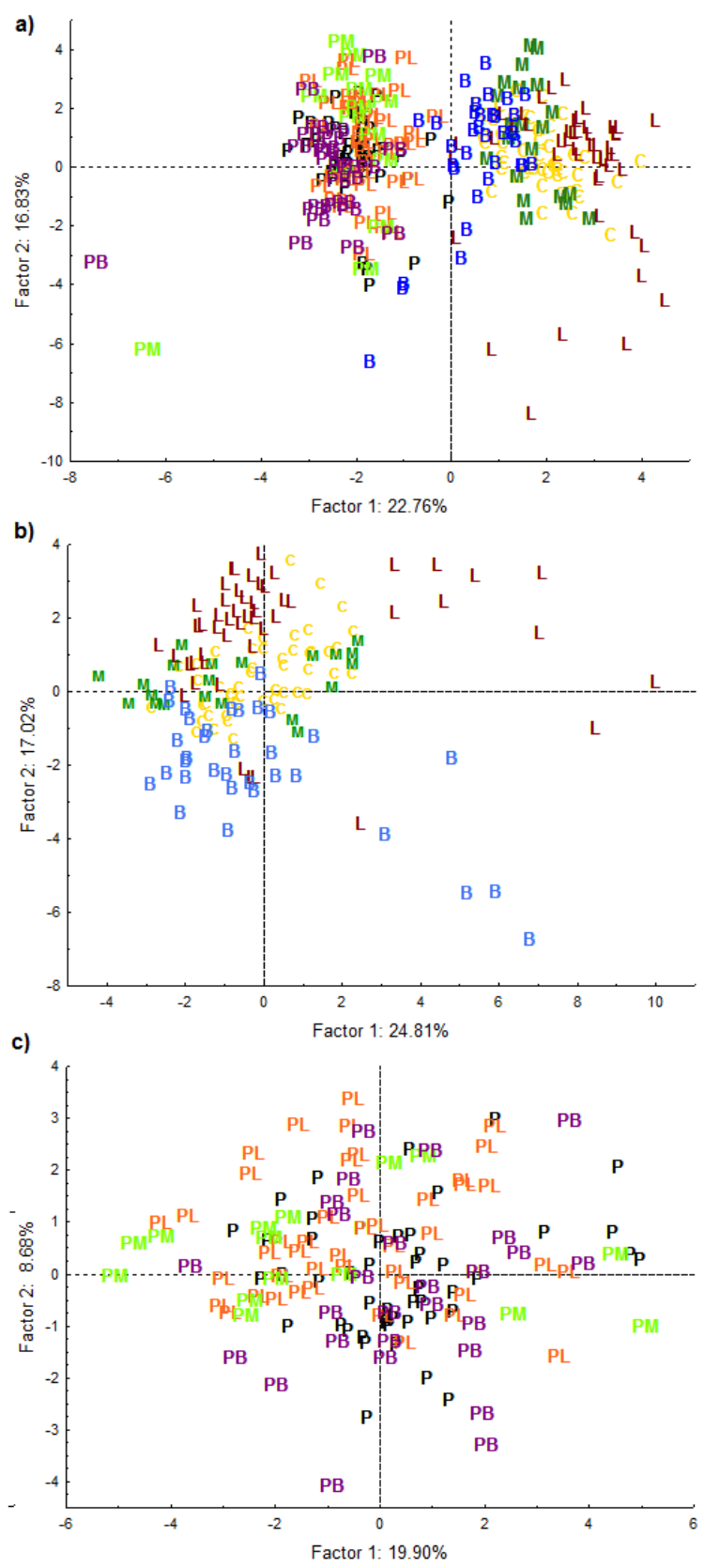

Figure 3. a) Simultaneous projection of the eight curimba treatments onto the first two axes of the principal components analysis. $b$ ) Individual projection of the four curimba treatments without piapara onto the first two axes of the principal components analysis. c) Individual projection of the four curimba treatments with piapara onto the first two axes of the principal components analysis. Each letter or letter combination indicates the space occupied by individual fish from one of the treatments $(C=$ control tank, $L=$ tank with logs, $M=$ tank with artificial macrophytes, $B=$ tank with both logs and artificial macrophytes, $P=$ tank with piapara, $P L=$ tank with piapara and logs, $P M=$ tank with piapara and artificial macrophytes and $P B=$ tank with piapara and both logs and artificial macrophytes). Sample size: $C=50$, $L=51, M=21, B=34, P=48, P L=46, P M=15, P B=27$.

The DCA performed for the eight treatments showed that all treatments differed between themselves $(p<0.05)$ and the squared Mahalanobis distances indicated how different they were from each other (Table 2). In relation to the control (C), the distance 
of P, PL, PM and PB treatments, were much larger than L, M and B distances. Therefore it is evident that the social enrichment effect was much larger than the structural, and when both types of enrichment were used together there was not a big difference related to the P tank. The squared Mahalanobis distances from the control were not related to the number of individuals in the tanks, which clearly shows that density did not affect the morphological traits (Fig. 4a). Furthermore, according to the DCA, the morphological traits most affected by treatments varied depending on the presence or absence of piapara (Table 3). When piapara were present, the traits most influenced were the relative height of caudal peduncle (RHCdP) and relative width of caudal peduncle (RWCdP). When piapara were absent, the most affected traits were the relative area of dorsal fin (RADsF), aspect ratio of anal fin (ARAnF) and relative area of pectoral fin (RAPtF).

Table 2. Squared Mahalanobis Distances between treatments. Highlighted in bold are the distances from each treatment compared to control.

\begin{tabular}{c|l|l|l|l|l|l|l|l}
\hline Treatment & \multicolumn{1}{|c|}{$\mathbf{C}$} & \multicolumn{1}{|c|}{$\mathbf{L}$} & \multicolumn{1}{|c|}{ M } & \multicolumn{1}{|c|}{ B } & P & PL & PM & PB \\
\hline $\mathbf{C}$ & 0.00001 & & & & & & & \\
$\mathbf{L}$ & $\mathbf{9 . 4 8 6 1 4}$ & 0.00001 & & & & & & \\
$\mathbf{M}$ & $\mathbf{1 0 . 9 8 9 5 0}$ & 6.05425 & 0.00001 & & & & & \\
$\mathbf{B}$ & $\mathbf{1 5 . 9 5 4 9 6}$ & 13.96630 & 10.86478 & 0.00001 & & & & \\
$\mathbf{P}$ & $\mathbf{6 3 . 3 3 0 5 6}$ & 57.76256 & 42.19287 & 43.62259 & 0.00001 & & & \\
PL & $\mathbf{5 4 . 2 2 4 6 1}$ & 47.92367 & 36.99225 & 32.90138 & 5.64027 & 0.00001 & & \\
PM & $\mathbf{6 2 . 3 8 8 1 2}$ & 54.43418 & 42.46321 & 36.99586 & 9.51183 & 3.21852 & 0.00001 & \\
PB & $\mathbf{6 0 . 8 4 0 7 8}$ & 58.15736 & 43.81637 & 35.84109 & 7.81064 & 3.68499 & 7.64126 & 0.00001 \\
\hline
\end{tabular}

Table 3. Discriminant analysis for the two sets of tank treatments with and without Piapara, with Wilks' lambda $=0.07040, F(63,394)=8.9793$ and $p<0.00001$ and Wilks' lambda $=0.20333, F(63,335)=3.7513$ and $p<0.00001$, respectively. Highlighted in bold are the traits that were significant for the discrimination among treatments.

\begin{tabular}{l|l|l|l|l}
\hline \multirow{2}{*}{ Trait } & \multicolumn{2}{|c|}{ Without piapara } & \multicolumn{2}{c}{ With piapara } \\
\cline { 2 - 5 } & $\mathrm{F}(3,132)$ & $\mathrm{P}$ value & $\mathrm{F}(3,112)$ & P value \\
\hline CI & 0.56147 & 0.641376 & $\mathbf{4 . 5 0 9 2 4 4}$ & $\mathbf{0 . 0 0 5 0 2 5}$ \\
IVF & 1.08495 & 0.357866 & 0.221322 & 0.881416 \\
RBH & $\mathbf{2 . 8 5 8 2 3}$ & $\mathbf{0 . 0 3 9 5 0 3}$ & $\mathbf{9 . 3 1 7 0 5 8}$ & $\mathbf{0 . 0 0 0 0 1 5}$ \\
REP & 0.45582 & 0.713624 & 0.893002 & 0.447202 \\
REA & 1.18988 & 0.316187 & 1.691071 & 0.172998 \\
RHeL & $\mathbf{3 . 0 0 8 4 0}$ & $\mathbf{0 . 0 3 2 6 1 7}$ & $\mathbf{6 . 2 7 9 5 1 6}$ & $\mathbf{0 . 0 0 0 5 6 1}$ \\
RLCdP & $\mathbf{3 . 6 6 7 1 5}$ & $\mathbf{0 . 0 1 4 0 5 5}$ & 1.129327 & 0.340394 \\
RHCdP & 0.48526 & 0.693099 & $\mathbf{3 . 7 7 9 0 1 8}$ & $\mathbf{0 . 0 1 2 5 8 3}$ \\
WCdP & 1.09736 & 0.352691 & $\mathbf{3 . 2 1 8 6 0 5}$ & $\mathbf{0 . 0 2 5 5 2 2}$ \\
CICdP & 0.78022 & 0.507005 & 2.622597 & 0.054132 \\
RADSF & $\mathbf{3 . 0 3 0 6 0}$ & $\mathbf{0 . 0 3 1 7 0 5}$ & 1.711437 & 0.168722 \\
RACdF & $\mathbf{1 3 . 5 8 0 6 7}$ & $\mathbf{0 . 0 0 0 0 0 0}$ & $\mathbf{3 . 9 3 2 1 3 7}$ & $\mathbf{0 . 0 1 0 3 7 5}$ \\
ARCdF & $\mathbf{4 . 7 4 5 3 2}$ & $\mathbf{0 . 0 0 3 5 5 3}$ & $\mathbf{5 . 9 0 5 8 6 3}$ & $\mathbf{0 . 0 0 0 8 8 7}$ \\
RAAnF & 2.29865 & 0.080410 & 0.237166 & 0.870283 \\
ARAnF & $\mathbf{3 . 2 8 4 5 9}$ & $\mathbf{0 . 0 2 2 9 2 0}$ & 0.701614 & 0.553013 \\
RLPIF & 1.16033 & 0.327458 & 1.624861 & 0.187627 \\
RAPIF & 2.34158 & 0.076162 & 1.558402 & 0.203494 \\
ARPIF & $\mathbf{8 . 0 6 2 2 3}$ & $\mathbf{0 . 0 0 0 0 5 7}$ & $\mathbf{4 . 8 8 2 9 6 5}$ & $\mathbf{0 . 0 0 3 1 4 9}$ \\
RLPtF & 1.03137 & 0.380968 & 2.618520 & 0.054411 \\
RAPtF & $\mathbf{1 6 . 2 7 4 7 3}$ & $\mathbf{0 . 0 0 0 0 0 0}$ & 1.946569 & 0.126179 \\
ARPtF & $\mathbf{3 . 0 8 5 1 5}$ & $\mathbf{0 . 0 2 9 5 7 2}$ & 0.615030 & 0.606661 \\
\hline
\end{tabular}


There was a significant difference between the centroid distances and, therefore, between the morphological variability among treatments. The curimba populations farmed together with piapara showed greater morphological variability than the populations farmed alone (Fig. 4b). Relative to both the control (C) and the treatments without piapara, all treatments with piapara generated significantly greater morphological variability, but did not differ among themselves. These results show that social enrichment (as opposed to structural enrichment) increased morphological variability.
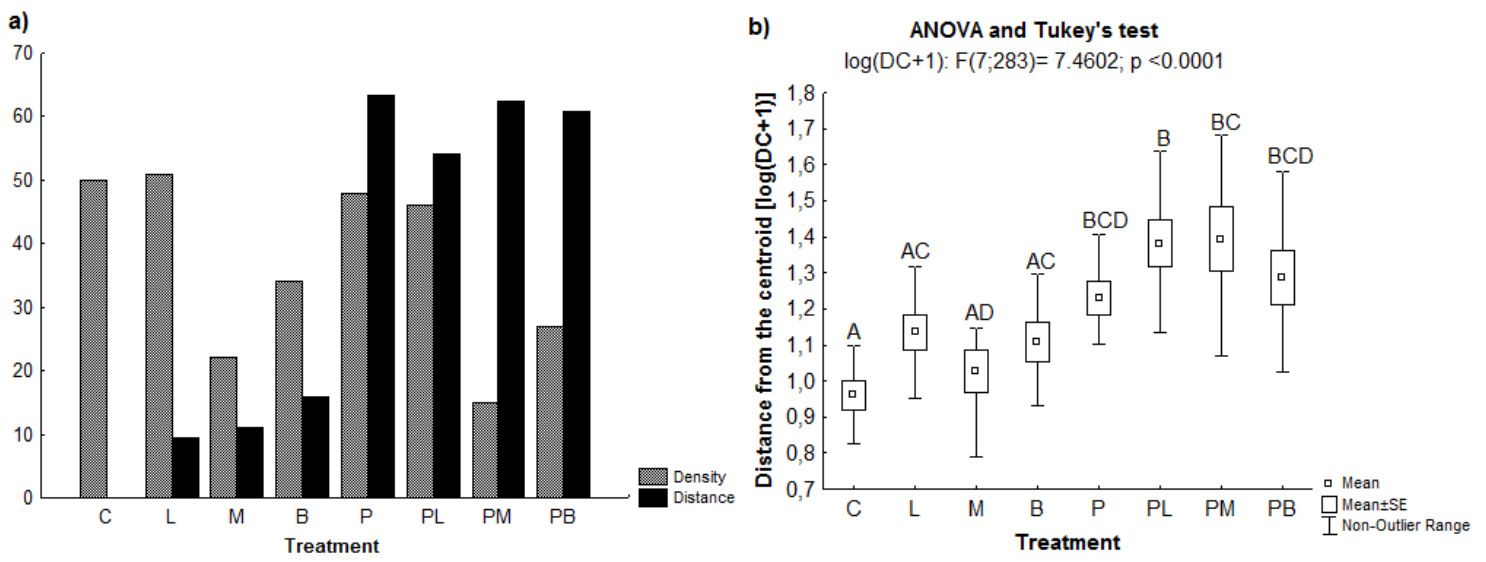

Figure 4. a) Densities of individuals per tank compared with the squared Mahalanobis distances of each treatment from the control $(C) . b)$ Log of the distance from the centroid plus one $[\log (D C+1)]$ for each curimba treatment population. After a significant ANOVA $(P<$ 0.05), Tukey's test compared the $\log (D C+1)$ values among the treatments $(C=$ control tank, $L=$ tank with logs, $M=$ tank with artificial macrophytes, $B=$ tank with both logs and artificial macrophytes, $P=$ tank with piapara, $P L=$ tank with piapara and logs, $P M=$ tank with piapara and artificial macrophytes and $P B=$ tank with piapara and both logs and artificial macrophytes). Treatments with the same letter above their ranges did not differ significantly. Sample size: $C=50, L=51, M=21, B=34, P=48, P L=46, P M=15, P B=27$.

\section{Discussion}

During interspecific competition, individuals from one species suffer a reduction in fertility, growth or survival as a result of resource exploitation or interference by another species (Begon et al, 2007). Intraspecific competition acts in a similar way but on individuals of the same species. In fish hatcheries, individuals compete for the same resources, such as food and space, and in the case of structured tanks, they may also compete for the added substrates. This competition may be intra and/or interspecific, and the two types of competition may interact, since the presence of another species may alter the interactions among individuals of the same species and vice versa (Persson and Greenberg, 1990).

In this study, the presence of piapara in the tanks did not directly affect curimba survival, however enrichment with artificial macrophytes had a negative effect (the number of curimba at the end of the experiment was lower in tanks enriched with macrophytes). A possible explanation is that foraging was impaired by the artificial macrophytes, because the plastic filaments tangled and/or trapped the food particles (personal observation), making them less accessible to fish. Tátrai and Herzig (1995) found similar result in an experiment with razor fish, which showed significantly 
reduced feeding levels in a complex environment with submerged macrophytes. They attribute the lower feeding efficiency to the macrophytes, that obstruct light, restrict predators in their movement and increase the chances for mobile invertebrates to escape from an attack. In another recent study, we found that artificial macrophytes hamper the development of curimba, decreasing the condition factor of individuals (Saraiva and Pompeu, 2014). However, in the present study, the condition factor of curimba in the tanks treated with macrophytes was not significantly affected, but this may be a result of the higher mortality and consequent lower density in these tanks. Curimba mortality was even higher in the tanks with both piapara and artificial macrophytes than in tanks with only artificial macrophytes. This result supports the explanation raised above, indicating an interaction between the effects of the two enrichment types: the tangled artificial macrophytes in addition to intra-specific competition with piapara led to a food resource limitation.

Similar to survival, curimba growth was not directly affected by the presence of piapara, since fish from tank $\mathrm{P}$ did not differ in size of those from the control (C). Standard length was significant bigger than the control only when both structural and social enrichment were present in tanks (PL, PM and PB). This result differs from the findings of Berejikian et al. (2001), who found that the growth of steelhead Oncorhynchus mykiss varied with the presence of competitors, but not with the structural enrichment of hatchery tanks. However, it is important to note that in PM and PB tanks, the density of curimba was lower, so greater growth was already expected (Maynard et al., 1994). The bigger fish size in these tanks is most likely related to the low density than to the enrichment. In the PL tank, despite the high population density and the presence of piapara, curimba growth rates were higher than those from control, indicating that the presence of logs favored growth. There was a considerable accumulation of periphyton on logs, which can favor fish growth and survival (vam Dam et al., 2002; Azim et al., 2005). However, enrichment with logs alone is not enough to explain the larger growth, since a significant increase in fish size did not occur in L tank. Thus, in some way, the presence of piapara must also have contributed to the higher standard length observed in the PL tank.

Logs also increased the condition factor in L and B tanks in relation to C. This improvement was certainly related to the periphyton on the logs, which worked as a supplementary food resource for curimba (personal observation). However, in the presence of piapara there was no increase, nor decrease, in curimba condition factor in any tank (P, PL, PM and PB). Thus, piapara apparently did not physiologically impair curimba (considering the high growth rates in the PL tank), yet the presence of piapara prevented improvements in curimba condition when logs were present, as occurred in L and B tanks. This finding may be evidence of exploitative competition, which occurs when the levels of a resource (in this case, periphyton on logs) decrease due to the presence and activity of other individuals or species (in this case piapara) (Begon et al., 2007).

Enrichment also affected morphological traits. Morphological divergence caused by competition has been described in some studies (Cussac et al., 1998; Olsson et al., 2006), however, it was not always possible to prove that the competition was the source of the morphological variation detected (Cussac et al., 1998). In the present study, by comparing tanks with and without piapara we were able to detect an effect of this species on the morphology of curimba. By comparing tanks without structures to those with different structure types, we could also demonstrate the structural enrichment 
effect, in the same way as in a previous study (Saraiva and Pompeu, 2014). Since the same pattern of results was found in both studies we can certify, despite the lack of replications, that the changes in fish morphology were a result of the treatment itself and not of a possible tank effect.

We also have to consider that fishes grow allometrically, so the body shape changes depending on the growth (Klingenberg and Froese, 1991). As fish from PL, PM and PB tanks were bigger than those from control tank, so it was expected that they had suffered the biggest mophological changes. But according to Mahalanobis distances, fish from P tank were as different as PL, PM and PB fish. So, if there is an allometric effect on morphology, it is added to the enrichment effect, which does not nullify it.

As shown above, both the social as the structural enrichment have affected fish morphology, but the Mahalanobis distances indicated that the social enrichment effect was much more stronger than the structural. Contrary to our expectations, the effect of one enrichment type did not add to the effect of the other, increasing fish divergence relative to the control. On the contrary, social enrichment seems to have diluted the effect of structural enrichment, as structuring ceased to be as important for curimba morphological variation when in the presence of piapara. One of the most important results of this study, it was that the social enrichment had increased the morphological variability in the reared curimba populations, approaching them of the wild populations (Taylor, 1986; Saraiva and Pompeu, 2016).

Upon analyzing the morphological traits that most contributed to differentiation among populations in DCA, we note that traits varied depending on the presence or absence of piapara. Relative length of caudal peduncle, relative area of dorsal fin, aspect ratio of anal fin, relative area of pectoral fin and aspect ratio of pectoral fin were only important in tanks without piapara. The first trait is linked to swimming ability (Gatz, 1979; Watson and Balon, 1984) and the others are related to performance of maneuvers (Gosline, 1971; Watson and Balon, 1984; Breda et al., 2005), suggesting that the curimba may have swum and used the structures more when piapara was absent. If so, we can assume that competition for space occurred and piapara dominated the structures at the expense of the curimba. However, in the absence of behavioral data is not possible affirm this.

Despite the logistic limitation of lacking replicates for each treatment, the results obtained indicate that fish raised with an interspecific competitor could present more varied morphology. Wild populations exhibit great variability in shape (Taylor, 1986; Saraiva and Pompeu, 2016). Therefore, simply using two or more species is a potential management tool in hatcheries to obtain fish more similar to wild ones. Structural enrichment could also lead to a distinct morphology, but to a lesser magnitude. Additional studies should be conducted to improve enrichment techniques, by analyzing behavioral data and identifying the most appropriate structures, as the structural enrichment effect will depend on the species, life stage and the enrichment type (Näslund and Johnsson, 2014)

Acknowledgements. We are most grateful to Companhia Energética de Minas Gerais (CEMIG) for the support and to everyone at the fish station of Volta Grande for essential help during this experiment, especially João de Magalhães Lopes, Alessandra Bedore and Sônia Maria Ramos. We are also grateful to Júlio Louzada for the valuable comments on the manuscript. PSP was awarded a researched productivity grant (CNPq 30402/2014-3) and a Minas Gerais scholarship grant (FAPEMIG PPM 608-15). 


\section{REFERENCES}

[1] Agostinho, A.A., Júlio-Junior, H.F. (1999): Peixes da bacia do Alto Paraná. Pp. 374-400. In: Lowe-McConnell, R.H. (Ed.). Estudos ecológicos de comunidades de peixes tropicais. Universidade de São Paulo, São Paulo.

[2] Agostinho, A.A., Gomes, L.C., Pelicice, F.M. (2007): Ecologia e manejo de recursos pesqueiros em reservatórios do Brasil. Universidade Estadual de Maringá, Maringá.

[3] Araki, H., Berejikian, B.A., Ford, M.J., Blouin, M.S. (2008): Fitness of hatchery-reared salmonids in the wild - Evolutionary Applications 1: 342-355.

[4] Azim, M.E., Verdegem, M.C.J., van Dam, A.A., Beveridge, M.C.M. (2005): Periphyton: ecology, exploitation and management. CABI Publishing, Wallingford.

[5] Batzina, A., Karakatsouli, N. (2012): The presence of substrate as a means of environmental enrichment in intensively reared gilthead seabream Sparus aurata: Growth and behavioral effects - Aquaculture 370-371: 54-60.

[6] Begon, M., Townsend, C.R., Harper, J.L. (2007): Ecologia de Indivíduos e Ecossistemas. Artmed, São Paulo.

[7] Belk, M.C., Benson, L.J., Rasmussen, J., Peck, S.L. (2008): Hatchery-induced morphological variation in an endangered fish: a challenge for hatchery-based recovery efforts - Canadian Journal of Fisheries and Aquatic Sciences 65: 401-408.

[8] Berejikian, B.A., Tezak, E.P., Riley, S.C., Larae, A.L. (2001): Competitive ability and social behavior of juvenile steelhead reared in enriched and conventional hatchery tanks and a stream environment - Journal of Fish Biology 59: 1600-1613.

[9] Berejikian, B.A., Tezak, E.P., Larae, A.L., Flagg, T.A., Kummerow, E., Mahnken, C.V.W. (2000): Social dominance, growth and habitat use of age-0 steelhead (Oncorhynchus mykiss) grown in enriched and conventional hatchery rearing environments - Canadian Journal of Fisheries and Aquatic Sciences 57: 628-636.

[10] Breda, L., Oliveira, E.F., Goulart, E. (2005): Ecomorfologia de locomoção de peixes com enfoque para espécies neotropicais - Acta Scientarium Biological Sciences 27: 371-381.

[11] Brown, C., Davidson, T., Laland, K. (2003): Environmental enrichment and prior experience of live prey improve foraging behaviour in hatchery-reared Atlantic salmon Journal of Fish Biology 63: 187-196.

[12] Cussac, V.E., Ruzzante, D., Walde, S., Macchi, P.J., Ojeda, V., Alonso, M.F., Denegri, M.A. (1998): Body shape variation of three species of Percichthys in relation to their coexistence in the Limay river basin, in northern Patagonia - Environmental Biology of Fishes 53: 143-153.

[13] Elliott, B.M., Grunberg, N.E. (2005): Effects of social and physical enrichment on open field activity differ in male and female Sprague-Dawley rats - Behavioural Brain Research 165: 187-196.

[14] Fleming, I.A., Jonsson, N.B., Gross, M.R. (1994): Phenotypic divergence of sea-ranched, farmed, and wild salmon - Canadian Journal of Fisheries and Aquatic Sciences 51: 28082824.

[15] Garduño-Paz, M.V., Couderc, S., Adams, C. E. (2010): Habitat complexity modulates phenotype expression through developmental plasticity in the threespine stickleback Biological Journal of the Linnean Society 100: 407-413.

[16] Gatz Junior, A.J. (1979): Ecological morphology of freshwater stream fishes - Tulanne Studies in Zoology Botany 21: 91-124.

[17] Gosline, W.A. (1971): Functional morphology and classification of teleostean fishes. University of Hawaii, Honolulu.

[18] Hard, J.J., Berejikian, B.A., Tezak, E.P., Schroder, S., Knudsen, C.M., Parker, L.T. (2000): Evidence for morphometric differentiation of wild and captively reared adult coho salmon: a geometric analysis - Environmental Biology of Fishes 58: 61-73. 
[19] Hora, S.L. (1930): Ecology, bionomics and evolution of the torrential fauna, with special reference to the organs of attachment - Philosophical Transactions of the Royal Society of London 28: 171-282.

[20] Klingenberg, C.P., Froese, R. (1991): A Mulltivariate Comparison of Allometric Growth Patterns - Systematic Zoology 40: 410-419.

[21] Leonardi, R., Buchanan-Smith, H.M., Dufour, V., MacDonald, C., Whiten, A. (2010): Living together: behavior and welfare in single and mixed species groups of capuchin (Cebus apella) and squirrel monkeys (Saimiri sciureus) - American Journal of Primatology 72: 33-47.

[22] Maynard, D.J., Flagg, T.A., Mahnken, C.V.W. (1994): A Review of Seminatural Culture Strategies for Enhancing the Postrelease Survival of Anadromous Salmonids. National Marine Fisheries Service, Seattle.

[23] Näslund, J., Johnsson, J.I. (2014): Environmental enrichment for fish in captive environments: Effects of physical structures and substrates - Fish and Fisheries 1-30.

[24] Newberry, R.C. (1995): Environmental enrichment: Increasing the biological relevance of captive environments - Applied Animal Behavior Science 44: 229-243.

[25] Olla, B.L., Davis, M.W., Ryer, C.H. (1998): Understanding how the hatchery environment represses or promotes the development of behavioral survival skills Bulletin of Marine Science 62: 531-550.

[26] Olsson, J., Svanba, R., Eklo, P. (2006): Growth rate constrain morphological divergence when driven by competition - Oikos 1: 15-22.

[27] Persson, L., Greenberg, L. (1990): Interspecific and intraspecific size class competition affecting resource use and growth of perch, Perca fluviatilis - Oikos 59: 97-106.

[28] Pouilly, M., Lino, F., Bretenoux, J.G., Rosales, C. (2003): Dietarymorphological relationships in a fish assemblage of the Bolivian Amazonian floodplain - Journal of Fish Biology 62: 1137-1158.

[29] Ricker, W.E. (1975): Computation and interpretation of biological statistics of fish populations. Fisheries and Marine Service, An Arbor.

[30] Roberts, L.J., Taylor, J., Garcia de Leaniz, C. (2011): Environmental enrichment reduces maladaptive risk-taking behavior in salmon reared for conservation - Biological Conservation 144: 1972-1979.

[31] Saraiva, S.O., Pompeu, P.S. (2014): The effect of structural enrichment in hatchery tanks on the morphology of two neotropical fish species - Neotropical Ichthyology 12: 891901.

[32] Saraiva, S. O., Pompeu, P. S. (2016): Fish hatchering and its effects on the morphology of Prochilodus lineatus (Actinopterygii: Prochilodontidae) - Brazilian Journal of Biology 76: 209-217.

[33] Schapiro, S.J., Bloomsmith, M.A., Suarez, S.A., Porter, L.M. (1996). Effects of social and inanimate enrichment on the behavior of yearling rhesus monkeys - American Journal of Primatology 40: 247-260.

[34] Solem, Ø., Berg, O.K., Kjøsnes, A.J. (2006): Inter- and intra-population morphological differences between wild and farmed Atlantic salmon juveniles - Journal of Fish Biology 69: 1466-1481.

[35] Swain, D.P., Riddell, B.E., Murray, C.B. (1991): Morphological differences between hatchery and wild populations of coho salmon (Oncorhynchus kisutch): environmental versus genetic origin - Canadian Journal of Fisheries and Aquatic Sciences 48: 17831791.

[36] Tátrai, I., Herzig, A. (1995): Effect of habitat structure on the feeding efficiency of young stages of razor fish (Pelecus cultratus (L.)): an experimental approach - Hydrobiologia 299: 75-81.

[37] Taylor, E.B. (1986): Differences in morphology between wild and hatchery populations of juvenile Coho Salmon - The Progressive Fish-Culturist 48: 171-176. 
[38] van Dam, A.A., Beveridge, M.C.M., Azim, M.E., Verdegem, M.C.J. (2002): The potential of fish production based on periphyton - Reviews in Fish Biology and Fisheries 12: $1-31$.

[39] Watson, D.J., Balon, E. (1984): Ecomorphological analysis of taxocenes in rainforest streams of northern Borneo - Journal of Fish Biology 25: 371-384.

[40] Winemiller, K.O. (1991): Ecomorphological diversification in lowland freshwater fish assemblages from five biotic regions - Ecological Monographs 61: 343-365. 